hep-th/0503061

\title{
Classification and a toolbox for orientifold models
}

\author{
P. Anastasopoulos ${ }^{1, \alpha, \beta}$, A. B. Hammou ${ }^{2, \alpha, \gamma}$ \\ ${ }^{\alpha}$ Department of Physics, University of Crete, \\ 71003 Heraklion, GREECE. \\ ${ }^{\beta}$ Laboratoire de Physique Théorique Ecole Polytechnique, \\ 91128 Palaiseau, FRANCE. \\ ${ }^{\gamma}$ Departement de Physique, Université des Sciences et Technologie d'Oran, \\ BP 1505, Oran, El M'Naouer, ALGERIE
}

\begin{abstract}
We provide the general tadpole conditions for a class of supersymmetric orientifold models by studing the general properties of the elements included in the orientifold group. In this talk, we concentrate on orientifold models of the type $T^{6} / Z_{M} \times Z_{N}$.
\end{abstract}

\footnotetext{
${ }^{1}$ panasta@physics.uoc.gr

2amine@physics.uoc.gr
} 


\section{Introduction}

Orientifolds are a generalization of orbifolds [1, 2, 3], where the orbifold symmetry includes orientation reversal on the worldsheet. The orientifold group contains elements of two kinds: internal symmetries of the worldsheet theory forming a group $G_{1}$ and elements of the form $\Omega \cdot g$, where $\Omega$ is the worldsheet parity transformation and $g$ is some symmetry element now taken from a group $G_{2}$. Closure implies that $\Omega \cdot g \cdot \Omega \cdot g^{\prime} \in G_{1}$ for $g, g^{\prime} \in$ $G_{2}$. The full orientifold group is $G_{1}+\Omega G_{2}$. The one loop amplitude implementing the $\Omega$ projection is interpreted as Klein Bottle amplitude and has in general ultraviolet divergences (tadpoles). These ultraviolet divergences are interpreted as sources in space time, that couple to the massless type IIB fields, the metric, the dilaton and the RR-forms. They are localized in sub-manifolds of space-time, known as Orientifold planes, $O_{p}$. These are non dynamical objects characterized by their charges and tensions. Consistency and stability of the theory are assured if D-branes are introduced in a way that guarantees the cancellation of these tadpoles. RR-tadpole cancellation is equivalent to the vanishing of gauge charge in a compact space, whereas NS-tadpole cancellation is equivalent to the vanishing of forces in the D-brane/O-plane vacuum configuration. In this talk we study the tadpole conditions for a class of orientifold groups of the type $G+\Omega G$ where $G$ an orbifold group. Consider the projection of type IIB string theory on $R^{4} \times T^{6}$ by the orientifold group $G+\Omega G$. The orbifold group $G$ acts on the coordinates $z^{i}$ of the 6-dimensional torus $T^{6}$ that we take to be factorized as $T_{1}^{2} \times T_{2}^{2} \times T_{3}^{2}$ as

$$
\alpha=e^{2 \pi i \sum_{i=1}^{3}\left(v_{\alpha}^{i} J^{i}+r_{i} \delta_{\alpha}^{i} P^{i}\right)},
$$

with $J^{i}$ and $P^{i}$ the generators of rotations and diagonal translation in each of the internal two torus $T_{i}^{2}$ with radius $r_{i}$. To preserve supersymmetry the $v_{\alpha}^{i}$ 's should satisfy the condition $\sum_{i} v_{\alpha}^{i}=0$.

In this talk we restrict ourself to the case of rotations preserving some supersymmetry, and refer to [4] for the non supersymmetric models including shifts. It is not difficult to be convinced that the most general rotation elements $\alpha$ preserving some supersymmetry are such that $v_{\alpha}=\left(v_{\alpha}^{1}, v_{\alpha}^{2}, v_{\alpha}^{3}\right)$ with say $v_{\alpha}^{3}=0$ or $v_{\alpha}^{3} \neq 0$. 

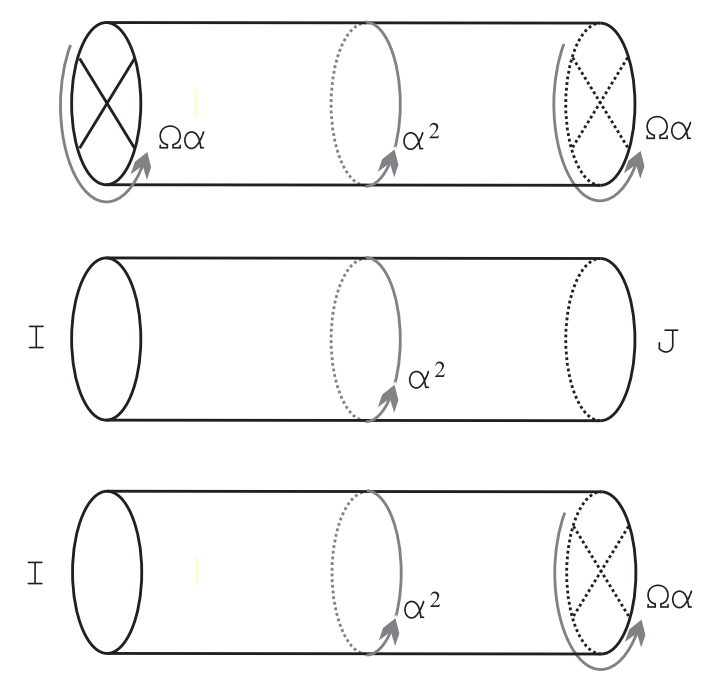

Figure 1: Klein-bottle, Annulus and Möbius strip. The one-loop amplitudes become tree-level in the transverse picture where an $\alpha^{2}$-twisted closed string propagates between crosscaps and boundaries.

\section{Supersymmetric Orientifolds}

\section{Klein Bottle}

Consider an element $\alpha$ of $G$. We can work out the contribution of this element to the Klein Bottle amplitude by using the trace formula:

$$
\mathcal{K}_{\alpha}=\operatorname{Tr}_{U+T}\left[\begin{array}{ll}
\Omega \alpha & \left.q^{L_{0}} \bar{q}^{\bar{L}_{0}}\right]
\end{array}\right.
$$

where the trace is over untwisted $U$ and twisted $T$ closed string states of the type IIB orbifold model considered. Due to the presence of $\Omega$ the only states contributing to $\mathcal{K}_{\alpha}$ are the untwisted states and the $Z_{2}$ twisted ones (by $Z_{2}$ we really mean order two elements i.e. $\left.R^{2}=1\right)$. The later exist only if the orbifold group $G$ contains $Z_{2}$ factors.

The contribution of an element $\alpha$ corresponds to a propagation of a closed string state projected out by $(\Omega \alpha)^{2}=\alpha^{2}$ and $(\Omega R \alpha)^{2}=\alpha^{2}$ when $R \in G$ since all elements of $G$ commute with $\Omega$ in our case.

To extract the massless tadpole contribution we perform a modular transformation $l=1 / 4 t$ where $t$ is the loop modulus and $l$ the cylinder length [5, 6] and then take the limit $l \rightarrow \infty$.

The general contribution to the tadpoles from Klein-Bottle amplitudes of a group 
element $\alpha$ such that $v_{\alpha}=\left(v_{\alpha}^{1}, v_{\alpha}^{2}, 0\right)$ is:

$$
\begin{aligned}
\left(1_{N S}-1_{R}\right) & \frac{1}{\prod_{l} 2 \sin 2 \pi v_{\alpha}^{l}}\left\{\sqrt{\mathcal{V}_{3}}\left(\prod_{l} 2 \cos \pi v_{\alpha}^{l}+\prod_{l} 2 \sin \pi v_{\alpha}^{l}\right)\right. \\
& \left.-\frac{1}{\sqrt{\mathcal{V}_{3}}} \sum_{i \neq j=1,2} \epsilon_{i j} 2 \cos \pi v_{\alpha}^{i} 2 \sin \pi v_{\alpha}^{j}\right\}^{2} .
\end{aligned}
$$

where $\epsilon_{12}=-\epsilon_{21}=1$. The first term corresponds to the contribution of $\Omega \alpha$, the second term is due to $\Omega R_{3} \alpha$ and both are proportional to the volume $\mathcal{V}_{3}$ of the third torus ${ }^{3}$. The remaining terms are the contribution from $\Omega R_{i} \alpha$ and are proportional to the inverse volume. The amplitude above is proportional to $\left(1_{N S}-1_{R}\right)$ and appears as perfect square [2, 8].

Consider now $v_{\alpha}=\left(v_{\alpha}^{1}, v_{\alpha}^{2}, v_{\alpha}^{3}\right)$ where $v_{\alpha}^{l=1,2,3} \neq 0$.

$$
\left(1_{N S}-1_{R}\right) \frac{1}{\prod_{l} 2 \sin 2 \pi v_{\alpha}^{l}}\left(\prod_{l} 2 \cos \pi v_{\alpha}^{l}+\sum_{i} 2 \cos \pi v_{\alpha}^{i} \prod_{l \neq i} 2 \sin \pi v_{\alpha}^{l}\right)^{2}
$$

The amplitude is again perfect square as it should [2, 8]. The different terms correspond as before to $\Omega \alpha$ and $\Omega R_{l} \alpha$ with $l=1,2,3$ respectively for $R_{l} \in G$.

In orientifold models tadpoles arise as divergences at the one-loop level, which are interpreted as inconsistencies in the field equations for the R-R potentials in the theory. These tadpoles can be regarded as the emission of an R-R closed string state from a Dpbrane (disc), a source of ( $\mathrm{p}+1)$-form $\mathrm{R}-\mathrm{R}$ potential and from an orientifold plane $\left(\mathbf{R} \mathbf{P}^{2}\right)$, which carries $\mathrm{R}-\mathrm{R}$ charges. The contributions we obtained above come from $O p$-planes sources of twisted $(\mathrm{p}+1)$-form $\mathrm{R}-\mathrm{R}$ potentials. For non trivial twists, the string zero modes vanish, and therefore the $D p$-brane is forced to sit at the fixed point. It simply means that the twisted $\mathrm{R}-\mathrm{R}$ potential can not propagate in peacetime.

\section{Annulus}

To cancel the aforementioned Klein-Bottle UV divergences -tadpoles- we need to add Dbranes to the spectrum (open string sector). For the model under consideration these are

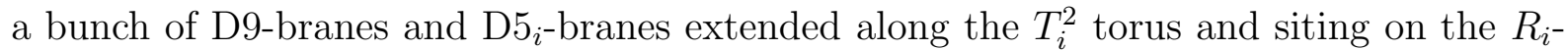
fixed points when the group $G$ contains $R_{i}$-factors. The Annulus amplitudes can be easily computed between all kinds of D-branes existing in the theory, with the contribution of the group element $\alpha$ given by the trace formula:

$$
\mathcal{A}_{I J, \alpha}=\operatorname{Tr}_{I J}\left[\alpha q^{L_{0}}\right]
$$

\footnotetext{
${ }^{3}$ By $R_{i}$ we denote a $Z_{2}$ rotation element that acts by reflecting the coordinates of the tori $T^{j}$ with $i \neq j$.
} 
where now the trace is over all open string states attached between $I$ and $J$ D-branes for $I, J=9,5_{i}$ siting at $\alpha$-fixed point. For simplicity we will always put the branes at one fixed point. To extract the tadpole contributions we need to perform a modular transformation to the transverse channel $l=1 / 2 t$ and then take the limit $l \rightarrow \infty$ [5].

For $\alpha$ such that: $v_{\alpha}=\left(v_{\alpha}^{1}, v_{\alpha}^{2}, 0\right)$ we have the following contribution to the tadpoles:

$$
\begin{gathered}
\left(1_{N S}-1_{R}\right) \frac{1}{\prod_{l} 2 \sin 2 \pi v_{\alpha}^{l}}\left[\sqrt{\mathcal{V}_{3}}\left(\operatorname{Tr}\left[\gamma_{\alpha, 9}\right]+\prod_{l} 2 \sin \pi v_{\alpha}^{l} \operatorname{Tr}\left[\gamma_{\alpha, 5_{3}}\right]\right)\right. \\
\left.-\frac{1}{\sqrt{\mathcal{V}_{3}}} \sum_{i \neq j=1,2} 2 \sin \pi v_{\alpha}^{j} \operatorname{Tr}\left[\gamma_{\alpha, 5_{i}}\right]\right]^{2} .
\end{gathered}
$$

The different terms correspond are the contribution to the tadpoles from the D-branes existing in the theory, and $\gamma_{\alpha, I}$ is the action of the group element $\alpha$ on the Chan-Paton factors in the $\mathrm{D} I$-brane sector. The amplitudes is again proportional to zero $\left(1_{N S}-1_{R}\right)$ reflecting the fact that the orientifold group action preserve some supersymmetry.

For $v_{\alpha}=\left(v_{\alpha}^{1}, v_{\alpha}^{2}, v_{\alpha}^{3}\right)$ the contribution to the tadpoles from the Annulus amplitudes is as follows:

$$
\left(1_{N S}-1_{R}\right) \frac{1}{\prod_{l} 2 \sin 2 \pi v_{\alpha}^{l}}\left(\operatorname{Tr}\left[\gamma_{\alpha, 9}\right]+\sum_{i=1}^{3} \prod_{l \neq i} 2 \sin \pi v_{\alpha}^{l} \operatorname{Tr}\left[\gamma_{\alpha, 5_{i}}\right]\right)^{2} .
$$

The structure of these amplitudes is similar to (4) without the volume dependance and with the product extended over $l=1,2,3$. It is explicitly perfect square as expected [2, 8].

\section{Möbius Strip}

Finally, the contribution of the group element $\alpha$ to the Möbius strip amplitude can be computed from the trace formula

$$
\mathcal{M}_{I, \alpha}=\operatorname{Tr}_{I}\left[\Omega \alpha q^{L_{0}}\right],
$$

where the trace is over open strings attached to DI-branes. To extract the contribution to the tadpoles we must perform a modular transformation to the transverse channel by $P=T S T^{2} S T$ where $T: \tau \rightarrow \tau+1$ and $S: \tau \rightarrow-1 / \tau, l=1 / 8 t$. Finally, we take the UV limit $l \rightarrow \infty$. The Möbius strip transverse channel amplitude is the mean value of the transverse channel Klein Bottle and Annulus amplitudes [2, 8. Taking into account this fact, extracting the UV limit in the Möbius strip amplitude and comparing with the amplitudes obtained in the Klein Bottle and Annulus gives constraints on the matrices $\gamma_{\alpha, I}$ and $\gamma_{\Omega . \alpha, I}$ :

$$
\operatorname{Tr}\left[\gamma_{\Omega \alpha, 9}^{T} \gamma_{\Omega \alpha, 9}^{-1}\right]=\operatorname{Tr}\left[\gamma_{\alpha^{2}, 9}\right],
$$




$$
\begin{aligned}
& \operatorname{Tr}\left[\gamma_{\Omega R_{i} \alpha, 9}^{T} \gamma_{\Omega R_{i} \alpha, 9}^{-1}\right]=-\operatorname{Tr}\left[\gamma_{\alpha^{2}, 9}\right], \\
& \operatorname{Tr}\left[\gamma_{\Omega \alpha, 5_{i}}^{T} \gamma_{\Omega \alpha, 5_{i}}^{-1}\right]=-\operatorname{Tr}\left[\gamma_{\alpha^{2}, 5_{i}}\right], \\
& \operatorname{Tr}\left[\gamma_{\Omega R_{i} \alpha, 5_{i}}^{T} \gamma_{\Omega R_{i} \alpha, 5_{i}}^{-1}\right]=\operatorname{Tr}\left[\gamma_{\alpha^{2}, 5_{i}}\right], \\
& \operatorname{Tr}\left[\gamma_{\Omega R_{j} \alpha, 5_{i}}^{T} \gamma_{\Omega R_{j} \alpha, 5_{i}}^{-1}\right]=-\operatorname{Tr}\left[\gamma_{\alpha^{2}, 5_{i}}\right],
\end{aligned}
$$

where in the last equation $i \neq j$ and $i, j=1,2,3$. These constraints apears to be the same for both $v_{\alpha}=\left(v_{\alpha}^{1}, v_{\alpha}^{2}, 0\right)$ or $v_{\alpha}=\left(v_{\alpha}^{1}, v_{\alpha}^{2}, v_{\alpha}^{3}\right)$.

\section{Tadpole conditions:}

The massless part of the transverse channel amplitude $\tilde{\mathcal{K}}_{\alpha}+\tilde{\mathcal{A}}_{\alpha}+\tilde{\mathcal{M}}_{\alpha}$ gives the tadpole conditions.

- $\alpha$ is such that $v_{\alpha}=\left(v_{\alpha}^{1}, v_{\alpha}^{2}, 0\right)$, then:

$$
\begin{aligned}
\operatorname{Tr}\left[\gamma_{\alpha^{2}, 9}\right]+4 \prod_{l} \sin 2 \pi v_{\alpha}^{l} \operatorname{Tr}\left[\gamma_{\alpha^{2}, 5_{3}}\right] & =32\left(\prod_{l} \cos \pi v_{\alpha}^{l}+\prod_{l} \sin \pi v_{\alpha}^{l}\right) \\
\sum_{i \neq j=1,2} 2 \sin 2 \pi v_{\alpha}^{j} \operatorname{Tr}\left[\gamma_{\alpha^{2}, 5_{i}}\right] & =32 \sum_{i \neq j=1,2} \epsilon_{i j} \cos \pi v_{\alpha}^{i} \sin \pi v_{\alpha}^{j} .
\end{aligned}
$$

The different terms exist when the corresponding $R$ factors do, as an example, if say $R_{3} \notin G$ then the second term on both sides of the first equation will be absent.

- $\alpha$ is such that $v_{\alpha}=\left(v_{\alpha}^{1}, v_{\alpha}^{2}, v_{\alpha}^{3}\right)$ then:

$$
\operatorname{Tr}\left[\gamma_{\alpha^{2}, 9}\right]+4 \sum_{i=1}^{3} \prod_{l \neq i} \sin 2 \pi v_{\alpha}^{l} \operatorname{Tr}\left[\gamma_{\alpha^{2}, 5_{i}}\right]=32\left(\prod_{l} \cos \pi v_{\alpha}^{l}+\sum_{i} \cos \pi v_{\alpha}^{i} \prod_{l \neq i} \sin \pi v_{\alpha}^{l}\right) .
$$

Note that in all cases the tadpole conditions holds for both NS and R sectors due to supersymmetry. On the other hand the tadpole condition for group elements which are not the square of some other element of $G$ (there is no element $\beta \in G$ such that $\alpha=\beta^{2}$ ), will receive contribution only from the Annulus amplitude. When this element is such that $v=\left(v_{\alpha}^{1}, v_{\alpha}^{2}, 0\right)$ or $v=g_{3}+v_{\alpha}$, the tadpole conditions will be the same as before, with zero on the right hand side. For the elements such that $v=g_{i}+v_{\alpha}$ the tadpole condition is not difficult to work out, leading to:

$$
\begin{aligned}
\operatorname{Tr}\left[\gamma_{R_{i} \alpha, 9}\right] & +4 \sin \pi v_{\alpha}^{i} \cos \pi v_{\alpha}^{j} \operatorname{Tr}\left[\gamma_{R_{i} \alpha, 5_{3}}\right] \\
& +2 \cos \pi v_{\alpha}^{j} \operatorname{Tr}\left[\gamma_{R_{i} \alpha, 5_{i}}\right]+2 \sin \pi v_{\alpha}^{i} \operatorname{Tr}\left[\gamma_{R_{i} \alpha, 5_{j}}\right]=0
\end{aligned}
$$

where $i \neq j=1,2$ and the different terms exist only if the corresponding $R_{i}$ factor does. If $v_{\alpha}=\left(v_{\alpha}^{1}, v_{\alpha}^{2}, v_{\alpha}^{3}\right)$, the tadpole conditions are the same as (9) without the right hand side (i.e. the right hand side is zero). 


\section{Some specific examples}

Let us discuss some of the examples that can be described by the general formulas we have obtained in the previous sections. The first example is the groups studied by Gimon and Johnson [6], where $G=Z_{N}$ for $N=2,3,4,6$ acting on $T^{4}$. The tadpole conditions are given by (8,9) with $v_{\alpha}^{1}=-v_{\alpha}^{2}=\frac{k}{N}, v_{\alpha}^{3}=0$ leading for odd $N$

$$
\operatorname{Tr}\left[\gamma_{2 k, 9}\right]=32 \cos ^{2} \frac{k}{N} \pi
$$

whereas for even $N$ :

$$
\begin{aligned}
& \operatorname{Tr}\left[\gamma_{2 k, 9}\right]-4 \sin ^{2} \frac{2 k}{N} \pi \operatorname{Tr}\left[\gamma_{2 k, 5_{3}}\right]=32 \cos \frac{2 k}{N} \pi \\
& \operatorname{Tr}\left[\gamma_{2 k-1,9}\right]-4 \sin ^{2} \frac{2 k-1}{N} \pi \operatorname{Tr}\left[\gamma_{2 k-1,5_{3}}\right]=0 .
\end{aligned}
$$

The tadpole conditions for the groups studied by Zwart [9], where $G=Z_{N} \times Z_{M}$, can be easily reproduced by using (8] 10). As an example the tadpole conditions for $Z_{N} \times Z_{M}$ where both $N$ and $M$ are odd are given by:

$$
\begin{aligned}
\operatorname{Tr}\left[\gamma_{2 k, 0,9}\right] & =32 \cos ^{2} \frac{k}{N} \pi \\
\operatorname{Tr}\left[\gamma_{0,2 l, 9}\right] & =32 \cos ^{2} \frac{l}{M} \pi \\
\operatorname{Tr}\left[\gamma_{2 k, 2 l, 9}\right] & =32 \cos \frac{k}{N} \pi \cos \frac{l}{M} \pi \cos \left(\frac{l}{M}-\frac{k}{N}\right) \pi .
\end{aligned}
$$

all the other cases can be easily worked out in a similar way. The tadpole conditions for the groups studied by Ibanez et al [10], where $G=Z_{N}$ with both $N$ even and odd and $G=Z_{N} \times Z_{M}$ with $N$ or $M$ even can be reproduce using (10) by specifying the vector $v_{\alpha}$ for the different group elements.

\section{Conclusion}

In this talk we have computed the tadpole conditions for the orientifold projection $G+\Omega G$ of type IIB string theory on $T^{6}$ in the supersymmetric case without specifying the orbifold group $G$. We have found complete agreement with the models studied in the literature. In this talk we have considered only the case when the D-branes are sitting on one fixed point mainly at the origin. However, it is not difficult to study more general situation where the D5-branes are distributed on different fixed points. We did not consider the additional freedom of adding Wilson lines, they are non dynamical. A non-trivial effective potential is generated for these gauge invariant operators that dynamically break part of the gauge group. Finally this analysis can be extended to more general orientifold groups $G_{1}+\Omega G_{2}$ 
by considering group elements that do not commute with $\Omega$ as well as asymmetric orbifold groups and to include fluxes which are T-dual to orientifold models with branes at angles.

\section{Acknowledgments}

The authors would like to thank Elias Kiritsis for very useful discussions. The work of A. B. Hammou was supported by RTN contracts HPRN-CT-2000-0131. This work was partially supported by RTN contracts INTAS grant, 03-51-6346, RTN contracts MRTNCT-2004-005104 and MRTN-CT-2004-503369 and by a European Union Excellence Grant, MEXT-CT-2003-509661. The work of P. Anastasopoulos was supported by a Herakleitos graduate fellowship.

\section{References}

[1] A. Sagnotti, arXiv:hep-th/0208020

[2] G. Pradisi and A. Sagnotti, Phys. Lett. B 216 (1989) 59.

[3] P. Horava, Nucl. Phys. B 327 (1989) 461; Phys. Lett. B 231 (1989) 251. D. Fioravanti, G. Pradisi and A. Sagnotti, Phys. Lett. B 321 (1994) 349 arXiv:hep-th/9311183;

[4] P. Anastasopoulos, A. B. Hammou, arXiv:hep-th/0503044.

[5] E. G. Gimon and J. Polchinski, Phys. Rev. D 54 (1996) 1667 arXiv:hep-th/9601038.

[6] E. G. Gimon and C. V. Johnson, Nucl. Phys. B 477 (1996) 715 arXiv:hep-th/9604129.

[7] M. Bianchi and A. Sagnotti, Nucl. Phys. B 361 (1991) 519.

[8] C. Angelantonj and A. Sagnotti, Phys. Rept. 371 (2002) 1 [Erratum-ibid. 376 (2003) 339] arXiv:hep-th/0204089.

[9] G. Zwart, Nucl. Phys. B 526 (1998) 378 arXiv:hep-th/9708040.

[10] G. Aldazabal, A. Font, L. E. Ibanez and G. Violero, Nucl. Phys. B 536 (1998) 29 arXiv:hep-th/9804026. 